\title{
Erratum to: Calculation of Parameters for Manufacturing the Bearing Surfaces by Pressurization
}

\author{
R. Sh. Gimadiev ${ }^{a}$, V. I. Khaliulin ${ }^{b}$, , and N. V. Levshonkov ${ }^{b}$
}

${ }^{a}$ Kazan State Power Engineering University, ul. Krasnosel'skaya 51, Kazan, 420066 Tatarstan, Russia

${ }^{b}$ Tupolev Kazan National Research Technical University, ul. Karla Marksa 10, Kazan, 420111 Tatarstan, Russia *e-mail: pla.kai@mail.ru

Received November 9, 2020

DOI: $10.3103 /$ S1068799820030241

The TeX presentation of formulas was updated in HTML file.

The original article can be found online at https://doi.org/10.3103/S1068799820020014. 